\title{
A NOTE ON SOBOLEV ALGEBRAS
}

\author{
ROBERT S. STRICHARTZ ${ }^{1}$
}

Abstract. Sufficient conditions are given for the Sobolev space $L_{v o}^{p}=\left\{f \in L^{p}\left(E^{n}\right): \mathcal{F}^{-1}(\hat{f}(\xi) w(\xi)) \in L^{p}\right\}$ to form an algebra under pointwise multiplication, when $1 \leqq p \leqq 2$. The conditions are verified for some examples.

In a previous work [6] we showed that the Sobolev space $L_{\alpha}^{p}$ $=\left\{f \in L^{p}\left(E^{n}\right): \mathcal{F}^{-1}\left(\hat{f}(\xi)\left(1+|\xi|^{2}\right)^{\alpha / 2}\right) \in L^{p}\right\}$ forms an algebra under pointwise multiplication for $1<p<\infty$ provided $\alpha>n / p$. The condition $\alpha>n / p$ is exactly what is required to have the containment $L_{\alpha}^{p} \subseteq L^{\infty}$. Thus it is reasonable to conjecture that for a wide class of weight functions $w(\xi)$ (replacing $\left(1+|\xi|^{2}\right)^{\alpha / 2}$ ), the space $L_{w}^{p}$ $=\left\{f \in L^{p}\left(E^{n}\right): \mathcal{F}^{-1}(\hat{f}(\xi) w(\xi)) \in L^{p}\right\}$ should form an algebra provided $L_{v}^{p} \subseteq L^{\infty}$. This has been established by Bagby [1] for weight functions of the form $w(\xi)=\left(1+i \xi_{1}+\sum_{j=2}^{n} \xi_{j}^{2}\right)^{\alpha}$ by generalizing our methods in [6]. The intricacy of his proof, however, indicates that other methods should be sought to handle more general weight functions, such as

$$
w(\xi)=\left(1+\sum_{j=1}^{n} \xi_{j}^{2 m_{j}}\right)^{\alpha}
$$

$m_{1}, \cdots, m_{n}$ positive integers, considered in Sadosky and Cotlar [4].

In this note we give such a method which works in the case $1 \leqq p$ $\leqq 2$. Since we require $L_{w}^{p} \subseteq A$, the space of Fourier transforms of $L^{1}$ functions, there does not seem to be an extension to $p>2$.

Denote by $M_{p}$ the space of $L^{p}$ multipliers; $M_{p}=\left\{m(\xi) \in L^{\infty}\left(E^{n}\right)\right.$ : $\mathcal{F}^{-1}(m(\xi) \hat{f}(\xi))$ is a bounded operator on $\left.L^{p}\right\}$ equipped with the operator norm.

ThEOREM. Let $1 \leqq p \leqq 2$. Assume

(a) $w^{-1} \in M_{p} \cap L^{p}$,

(b) $\sup _{\eta}\left\|w(\xi) /(w(\xi-\eta)+w(\eta)): M_{p}\right\|=M<\infty$.

Then $L_{w}^{p}$ is an algebra under pointwise multiplication.

Proof. Let $f, g \in L_{w}^{p}$. We must show that $\mathfrak{F}^{-1}(\hat{f} * \hat{g}(\xi) w(\xi)) \in L^{p}$. If we write $a(\xi, \eta)=w(\xi) /(w(\xi-\eta)+w(\eta))$ we have

Received by the editors July 30,1970 .

AMS 1970 subject classifications. Primary 46E35.

Key words and phrases. Sobolev spaces, $L^{p}$ multipliers, Sobolev algebras.

1 Research supported by NSF grant GP 22820. 


$$
\begin{aligned}
\mathcal{F}^{-1}(\hat{f} * \hat{g}(\xi) w(\xi))= & \mathcal{F}^{-1}\left(\int \hat{f}(\xi-\eta) \hat{g}(\eta) a(\xi, \eta) w(\eta) d \eta\right) \\
& +\mathcal{F}^{-1}\left(\int \hat{f}(\xi-\eta) \hat{g}(\eta) a(\xi, \eta) w(\xi-\eta) d \eta\right) .
\end{aligned}
$$

We show that each term separately is in $L^{p}$.

Note that $w^{-1} \in L^{p}$ implies trivially that $L_{w}^{p} \subseteq A$, in other words $\hat{f}, \hat{g} \in L^{1}$. Thus

$$
\begin{aligned}
&\left\|\mathfrak{F}^{-1}\left(\int \hat{f}(\xi-\eta) \hat{g}(\eta) a(\xi, \eta) w(\xi-\eta) d \eta\right)\right\|_{p} \\
& \leqq \int\left\|F^{-1}(\hat{f}(\xi-\eta) w(\xi-\eta) a(\xi, \eta))\right\|_{p}|\hat{g}(\eta)| d \eta \\
& \leqq M\left\|f: L_{w}^{p}\right\|\|\hat{g}\|_{1}
\end{aligned}
$$

by assumption (b). To handle the other term in (2) we make a change of variable $\eta \rightarrow \xi-\eta$ and repeat the argument interchanging $f$ and $g$. Q.E.D.

Let us show that all functions of the form (1) with $\alpha>0$ satisfy condition (b). Condition (a) is satisfied provided $\alpha>(2 p)^{-1} \sum_{j=1}^{n} m_{j}^{-1}$ (see [4]), when $L_{v o}^{p} \subseteq L^{\infty}$.

By the Marcinkiewicz multiplier theorem (see [2], [3], or [5]) (b) will follow if we can show

$$
\left|\xi^{\alpha} D_{\xi}^{\alpha} a(\xi, \eta)\right| \leqq M
$$

for all multi-indices $\alpha=\left(\alpha_{1}, \cdots, \alpha_{n}\right)$ such that $\alpha_{j}=0$ or 1 . This may be established by straightforward but tedious estimates using the elementary inequality

$$
\sum_{j=1}^{n} \xi_{j}^{2 m_{j}} \leqq M \max \left\{\sum_{j=1}^{n}\left(\xi_{j}-\eta_{j}\right)^{2 m_{j}}, \sum_{j=1}^{n} \eta_{j}^{2 m_{j}}\right\}
$$

if $M \geqq 2^{2 m_{j}+1}, j=1, \cdots, n$.

\section{REFERENCES}

1. R. Bagby, Lebesgue spaces of parabolic potentials, Illinois J. Math. (to appear).

2. P. Krée, Sur les multiplicateurs dans $\mathcal{F} L^{p}$, Ann. Inst. Fourier (Grenoble) 16 (1966), fasc. 2, 31-89. MR 35 \#7079.

3. W. Littman, C. McCarthy and N. Rivière, $L^{p-m u l t i p l i e r ~ t h e o r e m s, ~ S t u d i a ~ M a t h . ~}$ 30 (1968), 193-217. MR 37 \#6681. 
4. C. Sadosky and M. Cotlar, On quasi-homogeneous Bessel potential operators, Proc. Sympos. Pure Math., vol. 10, Amer. Math. Soc., Providence, R.I., 1967, pp. 275-287.

5. E. M. Stein, Intégrales singulières et fonctions differentiable de plusieurs variables, Lecture Notes, Faculté des Sciences d'Orsay, 1967.

6. R. S. Strichartz, Multipliers on fractional Sobolev spaces, J. Math. Mech. 16 (1967), 1031-1060. MR 35 \#5927.

Cornell University, Ithaca, New York 14850 